\title{
Clinical validation of Xpert MTB/RIF for the diagnosis of extrapulmonary tuberculosis
}

\author{
Enrico Tortoli*, Cristina Russo ${ }^{\#}$, Claudio Piersimoni ${ }^{\top}$, Ester Mazzola+, \\ Paola Dal Monte ${ }^{\S}$, Michela Pascarella ${ }^{f}$, Emanuele Borroni*, Alessandra Mondo**,

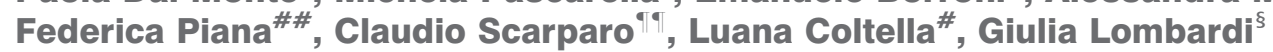 \\ and Daniela M. Cirillo*
}

ABSTRACT: Extrapulmonary tuberculosis (EPTB) accounts for more than $20 \%$ of tuberculosis (TB) cases. Xpert MTB/RIF (Xpert) (Cepheid, Sunnyvale, CA, USA) is a fully automated amplification system, for which excellent results in the diagnosis of pulmonary TB in highly endemic countries have been recently reported. We aimed to assess the performance of the Xpert system in diagnosing EPTB in a low incidence setting.

We investigated with Xpert a large number of consecutive extrapulmonary clinical specimens $(1,476$, corresponding to 1,068 patients) including both paediatric (494) and adult samples.

We found, in comparison with a reference standard consisting of combination of culture and clinical diagnosis of TB, an overall sensitivity and specificity of $\mathbf{8 1 . 3 \%}$ and $\mathbf{9 9 . 8 \%}$ for Xpert, while the sensitivity of microscopy was $48 \%$. For biopsies, urines, pus and cerebrospinal fluids the sensitivity exceeded $\mathbf{8 5} \%$, while it was slightly under $\mathbf{8 0} \%$ for gastric aspirates. It was, in contrast, lower than $50 \%$ for cavitary fluids. High sensitivity and specificity ( $86.9 \%$ and $99.7 \%$, respectively) were also obtained for paediatric specimens.

Although the role of culture remains central in the microbiological diagnosis of EPTB, the sensitivity of Xpert in rapidly diagnosing the disease makes it a much better choice compared to smear microscopy. The ability to rule out the disease still remains suboptimal.

KEYWORDS: Clinical validation, diagnostic accuracy, extrapulmonary tuberculosis, nucleic acid amplification, Xpert MTB/RIF

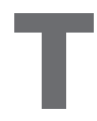
he proportion of extrapulmonary tuberculosis (EPTB) is quite high in industrialised countries. In the USA it is steadily increasing and according to the last available data (from 2005) it accounted for $21 \%$ of all tuberculosis (TB) cases [1]. In 2009, in European Union countries (including Italy and excluding the five World Health Organization (WHO)Euro high-priority countries), a quarter of $\mathrm{TB}$ cases $(24.4 \%)$ were extrapulmonary and a further $4.9 \%$ of patients had both pulmonary and extrapulmonary disease [2]. In contrast to pulmonary $\mathrm{TB}$, the diagnosis of EPTB is still a serious problem in industrialised countries, where it remains undetected for a long time in a considerable number of cases. A major hindrance to the diagnosis of EPTB is the atypical presentation, often simulating neoplasia and/or inflammatory disorders. Furthermore in the nonrespiratory specimens the bacterial load is generally very low, therefore strongly affecting the sensitivity of rapid tests such as acid-fast microscopy and nucleic acid amplification (NAT).
The large majority of commercially available NATs are validated for pulmonary samples only and the Centers for Disease Control and Prevention (CDC), even in their most recent recommendations, consider published data insufficient to promote their use in routine diagnostic testing [3].

Xpert MTB/RIF (Xpert) (Cepheid, Sunnyvale, CA, USA), a fully automated real time heminested PCR system implementing molecular beacon technology for the diagnosis of pulmonary TB infection, has been recently endorsed by the Scientific and Technical Advisory Board of the WHO as the most sensitive fast test for TB diagnosis in paucibacillary respiratory samples [4]. The test is currently recommended as a "first line" fast diagnostic test in endemic countries when rapid results are crucial for TB diagnosis in HIV-infected patients or for appropriate management of multidrug-resistant TB cases. Recent papers have investigated the capacity of Xpert assay to diagnose pulmonary $\mathrm{TB}$ in high burden TB countries $[5,6]$. In this study, we have

\section{AFFILIATIONS}

*Emerging Bacterial Pathogens Unit, San Raffaele Scientific Institute, and ${ }^{+}$Microbiology Laboratory, Niguarda Hospital, Milan,

\# Microbiology Laboratory, Bambino Gesù Paediatric Hospital, Rome,

-Regional Reference Mycobacteria Laboratory, Clinical Pathology Laboratory, United Hospitals, Ancona,

${ }^{\S}$ Microbiology Laboratory, Sant'Orsola University Hospital, Bologna,

${ }^{f}$ Microbiology Laboratory, San Bortolo Hospital, Vicenza,

**Microbiology Laboratory, Amedeo di Savoia Hospital, Turin,

\#\# Microbiology Unit, Santa Croce e

Carle Hospital, Cuneo, and

"Microbiology Laboratory,

Misericordia Hospital, Udine, Italy.

CORRESPONDENCE

E. Tortoli

Emerging Bacterial Pathogens Unit, San Raffaele Scientific Institute, via Olgettina 58, 20132 Milan Italy

E-mail e.tortoli@libero.it

Received:

Oct 112011

Accepted after revision:

Nov 242011

First published online:

Jan 122012

European Respiratory Journa

Print ISSN 0903-1936

Online ISSN 1399-3003 
evaluated the performance of the Xpert system on high number of different extrapulmonary specimens in a country with low TB incidence. The large collection of data allowed as well the assessment of microscopy and culture performance in extrapulmonary samples.

\section{MATERIALS AND METHODS}

Eight Italian laboratories contributed to the survey by providing retrospective results of a number of consecutive extrapulmonary specimens accepted for the diagnosis of EPTB. Xpert system was already in use in five laboratories; in the other three it was added to the routine workflow for TB diagnosis. Personnel involved in performing and reporting of Xpert were blind to the results of microscopy and cultures. All the contributing centres are accredited by the Italian Ministry of Health for laboratory diagnosis (including molecular tests) of TB. The study was approved by the ethics committee of the lead institution of this study (San Raffaele Scientific Institute, Milan, Italy).

The number of samples processed in each laboratory ranged from 34 to 376 , for a total of 1,493 corresponding to 1,068 patients. The specimens consisted mainly of biopsies, pleural fluids and gastric aspirates but a significant number of samples representative of each form of EPTB was included in the study (table 1). Body fluids other than pleural and cerebrospinal fluid (CSF) were classified as cavitary fluids. Gastric aspirates were also included in the study; although normally used to diagnose pulmonary TB, they differ substantially from sputum and their yield in Xpert test is poorly known.

Of the 1,476 samples with a valid result, 494 were collected from paediatric patients (age range 0-18 yrs).

A different pre-treatment was adopted according to the sample type; non-sterile samples were decontaminated with standard NALC NaOH (1\% final concentration) procedure and concentrated by centrifugation [7], while for sterile samples, only mechanical homogenisation was performed (if needed) before resuspension in saline and concentration.

On all the specimens, acid-fast microscopy (auramine-rhodamine staining) and culture, both in solid (Lowenstein-Jensen) and liquid (MGIT 960; Becton Dickinson Biosciences, Sparks, MD, USA) media were performed. The mycobacteria grown in culture were identified using commercially available DNA probe assays. Phenotypic susceptibility testing was performed by the "gold standard" automatic MGIT 960 SIRE (Becton Dickinson).

Only samples for which at least $0.5 \mathrm{~mL}$ of concentrated specimen was available for Xpert test were included in the study. Samples providing "indeterminate" Xpert results ("error", "invalid" or "no result"), were not re-tested. The protocol suggested by the producer for sample preparation and testing of non-respiratory samples was strictly followed.

Xpert results were compared with culture results. For patients whose samples were Xpert-positive and culture-negative, medical records were examined for clinical diagnosis of TB $[8,9]$. Patients with radiological and/or histological signs suggesting $\mathrm{TB}$ were considered $\mathrm{TB}$ cases only if a clinical improvement after anti-TB treatment was recorded. Patients

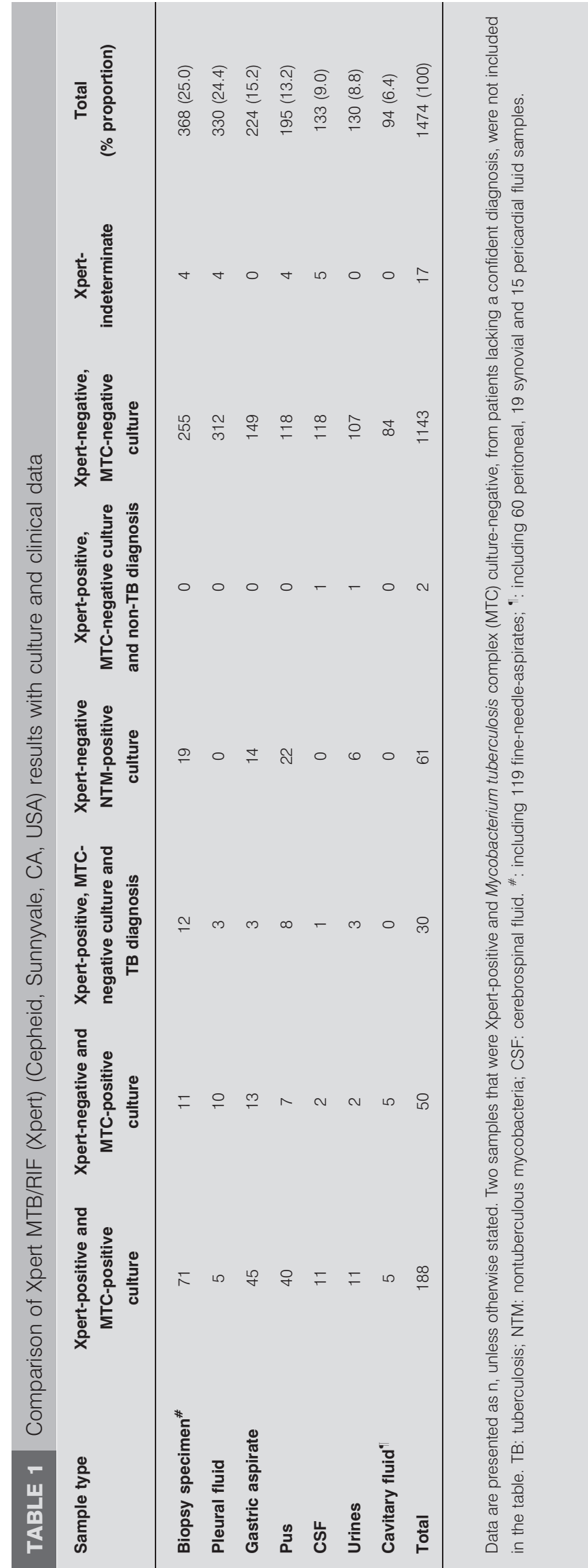


not treated for $\mathrm{TB}$, or not presenting evident improvement after treatment, were not considered TB cases.

Statistical analysis included the determination of specificity, sensitivity, and positive and negative likelihood ratios. The likelihood ratios were preferred to positive and negative predictive values as, differently from the latter, are not affected by prevalence [10]. Comparison between categorical variables was made using the Fisher's test; a p-value $<0.05$ was considered statistically significant.

\section{RESULTS}

The tests results were quite homogeneous and no significant variation in performance was observed across the different centres participating to the study.

In a very small proportion of samples $(17 ; 1.1 \%)$, indeterminate Xpert results were obtained.

In 128 samples (116 culture-positive) acid-fast bacilli were detected by microscopy while in 183 smear-negative samples, growth of mycobacteria was obtained in culture. In 61 culturepositive samples (18 of which were also smear-positive) a nontuberculous mycobacterium (NTM) was identified.

The sensitivity of microscopy was $48 \%$, when compared with culture, and $47.0 \%$, when compared to Xpert. Among the samples examined (including the culture-positives ones and those with clinical diagnosis), the sensitivity of culture was $81.3 \%$.

Out of 238 samples positive in culture for Mycobacterium tuberculosis complex (MTC), Xpert was positive in 188 and negative in 50 (table 1). 34 MTC culture-negative samples scored positive with Xpert; for 30 of the enrolled patients from which they had been collected, TB was clinically confirmed while, for two, it was ruled out. The remaining two, lost to follow-up and therefore lacking a confident diagnosis, were only included in the analysis comparing Xpert to culture results.

Overall sensitivity and specificity of Xpert were 79.0 and $97.3 \%$ respectively when compared to culture; these rose to 81.3 and $99.8 \%$ once discrepancies were resolved by considering the clinical diagnosis (table 2).

Separate analysis of the paediatric samples revealed (table 2) higher sensitivity and specificity values in comparison with the ones originated from adults.

As expected, the sensitivity in smear-negative samples was significantly lower than in smear-positive samples $(p<0.0001)$, but the specificity was higher (table 2).

When considering the yield of different clinical specimens, a sensitivity $>85 \%$ was obtained with CSF, biopsies, urines, pus samples and fine-needle aspirates while it was very poor for the body fluids (table 2). The specificity was excellent for all the types of samples examined, and ranged from 99.1 to $100 \%$; none of the samples growing NTMs in culture scored positive in Xpert assay.

Xpert detected rifampin resistance only in seven patients; all results were in full agreement with phenotypic drug susceptibility testing performed by the automatic MGIT 960.
Xpert test also provides a semi-quantitative report of the number of DNA copies detected in the sample; as expected it was "very low", or "low", in the large majority (78.4\%) of the samples that scored positive.

\section{DISCUSSION}

This is the first assessment of Xpert performance conducted on a high number of samples, from adult and paediatric patients, in a non-TB-endemic country.

A fair comparison with two previous studies investigating the performance of Xpert on extrapulmonary clinical samples is not possible because of major differences in study design. The first study, similar to ours for the epidemiological background, omitted in the evaluation the clinical diagnosis [11], while the other was carried out in a high prevalence TB country [12]. In addition, both studies did not target the paediatric population. When considering the overall sensitivity (fig. 1) and specificity, our results did not differ significantly either from the ones obtained by VADWAI et al. [12], in their survey in India $(p=0.36)$ or from those reported by HillemanN et al. [11], in Germany $(p=0.53)$. Diagnostic accuracy values better than ours were reported for body fluids and pus, in the first study, and for urine and gastric aspirates, in the second. In contrast, the diagnostic accuracy of our results was higher for biopsies and CSF compared to both the aforementioned studies.

In a third paper, excellent Xpert results (95\% sensitivity and $100 \%$ specificity) have been reported for 340 extrapulmonary specimens; however, separate analysis for different specimen types was not provided [13].

A very recent paper evaluated Xpert's ability in diagnosing tuberculous lymphadenitis from 50 fine needle aspirates and reported $96.7 \%$ sensitivity and $88.9 \%$ specificity [14]. On 119 needle aspirates, our sensitivity was significantly lower (84.8\%) but specificity was $100 \%$.

For what concerns data obtained with extrapulmonary specimens using commercial NATs other than Xpert, only two studies investigated large numbers of samples [15, 16]; similar to the present study, in both studies molecular results were compared to a gold standard combining both culture results and clinical diagnosis. The first evaluated the AMTD (GenProbe, San Diego, CA, USA) on 682 specimens [15], while in the second 918 samples were assayed by the ProbeTec ET (Becton Dickinson) [16]. Both reported very good results with important categories of specimens such as CSFs, gastric aspirates and tissue samples and for both the overall sensitivity was high $(89.3 \%$ and $82.7 \%$, respectively). In the paper by LARAQUE et al. [15], however, the high sensitivity was counterbalanced by very poor specificity $(74.5 \%)$.

There is a large number of studies evaluating Xpert on pulmonary specimens [5, 6, 17-23]; the sensitivity varies from $95 \%$ to $100 \%$ in smear-positive sputa, and from $47 \%$ to $77 \%$ in smear-negative ones. Our data score high within these ranges, despite the well-known lower yield of extrapulmonary samples in term of sensitivity.

A substantial proportion of tests $(494 ; 33.5 \%)$ were performed on paediatric samples. In this subgroup sensitivity and specificity $(86.9 \%$ and $99.7 \%$, respectively) were even higher than those in adults (difference not statistically significant; $\mathrm{p}=0.1$ ). 


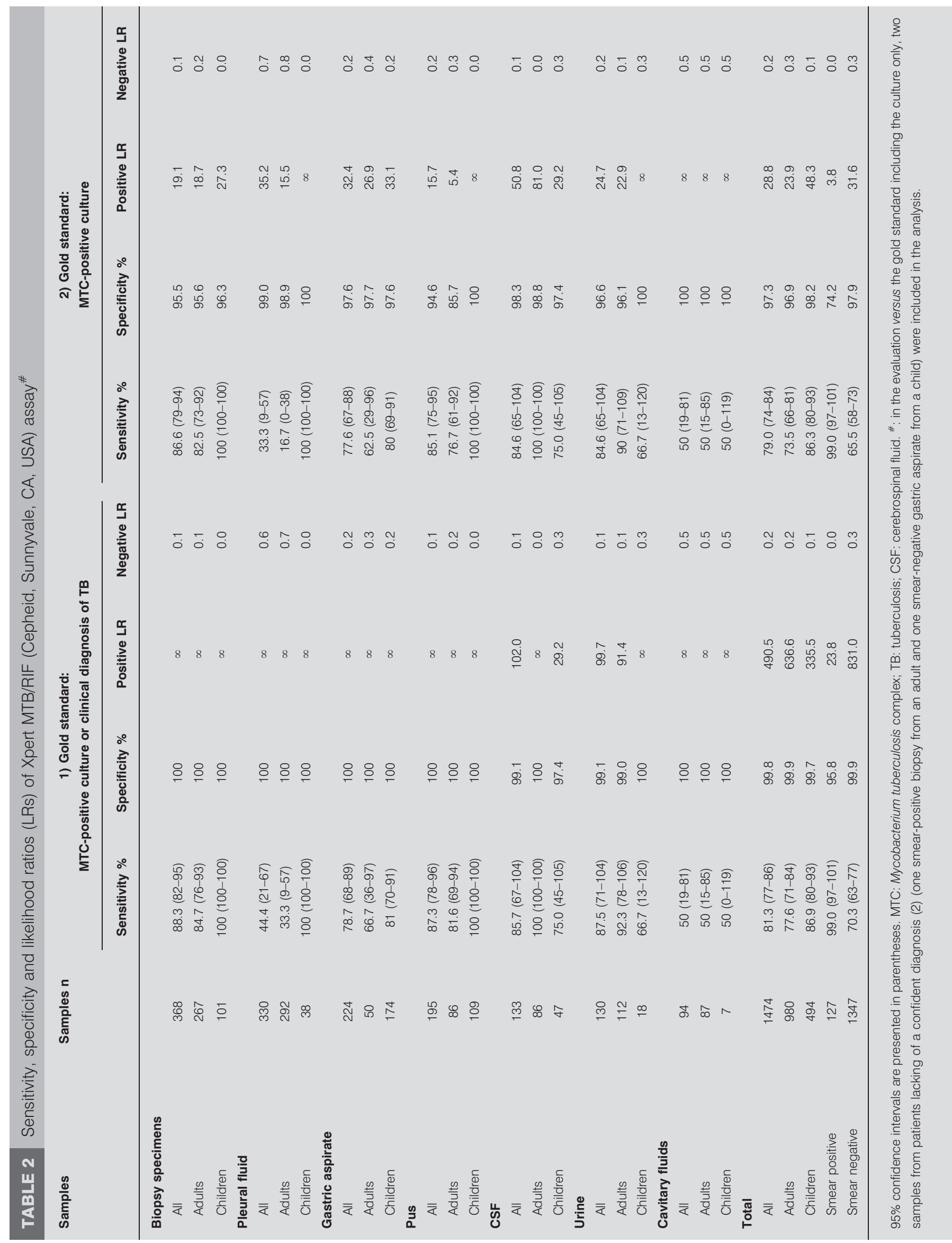




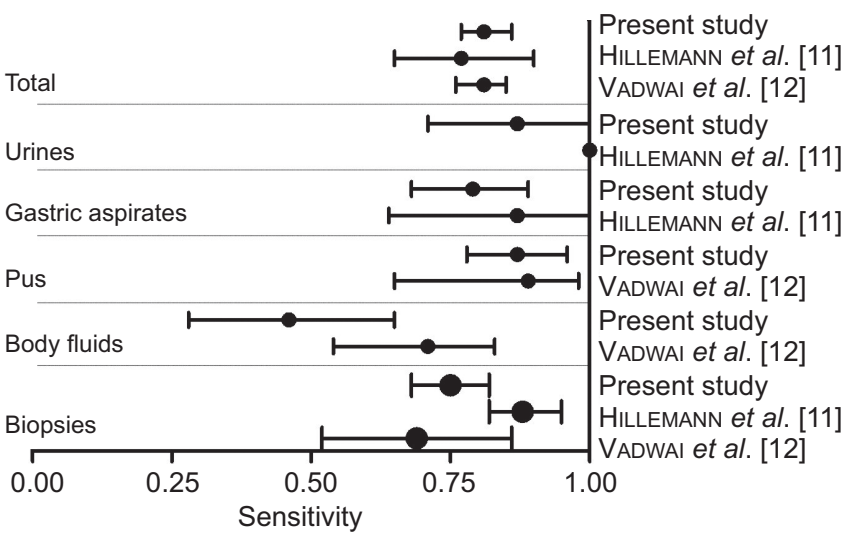

FIGURE 1. Forest plot comparing sensitivity estimates for Mycobacterium tuberculosis complex detection in different types of extrapulmonary samples. Three studies, including the present: only specimen types represented by at least 25 samples have been included.

The somehow unexpected better sensitivity obtained in paediatric specimens in comparison with adult ones is most likely due to the different distribution of low-yield materials (pleural and other cavitary fluids) in the two groups; they accounted in fact for $9.1 \%$ in children and for $38.7 \%$ in adults.

In the only paper targeting paediatric specimens, induced sputa were investigated for the diagnosis of pulmonary TB [22]; the results are therefore not comparable to ours. In this study, the only specimens aiming to diagnose pulmonary TB in children were gastric aspirates $(n=174)$. The sensitivity was $88 \%$, not substantially different from the one reported by NICOL et al. [22] (74.3\%).

Of the two false-positive Xpert results, one was obtained on a urine sample collected from a patient with bladder cancer. A positive Xpert result due to intravescical instillation of bacillus Calmette-Guérin cannot be excluded, as the patient had been cared for in a hospital implementing the immunotherapy for treatment of bladder cancer. Unfortunately the patient record was not available to confirm this hypothesis. The alternative explanation for this false-positive result, and for the other (concerning a CSF of a child for whom the TB was excluded) remains crosscontamination, despite the potentially contamination-free technology on which Xpert system relies upon.

As reported by others [12] it is possible that the decontamination step, required for performing the culture of non-sterile samples, has determined the lowering of bacillary load and consequently the reduction of test sensitivity.

Out of the scope of this study was the evaluation of the performance of Xpert to detect rifampin resistance, because of low prevalence of rifampin resistant TB in our epidemiological setting. Conventional susceptibility testing confirmed, however, the seven cases of rifampin resistance revealed by Xpert.

The diagnosis of $\mathrm{TB}$, and especially of $\mathrm{EPTB}$, is based on the combination of multiple tests; among them, the main role of the culture is once more confirmed with the potential of Xpert being high in ruling in, but suboptimal in ruling out, EPTB. Among the rapid tests investigated here, Xpert's sensitivity scored twice as high in comparison with microscopy thus doubling the proportion of rapid diagnoses, with important rebound on the patients' outcome.

\section{SUPPORT STATEMENT}

This work was partially supported by the European Community's Seventh Framework Programme (FP7-223681 to D.M. Cirillo).

\section{STATEMENT OF INTEREST}

A statement of interest for this study can be found at www.erj. ersjournals.com/site/misc/statements.xhtml

\section{ACKNOWLEDGEMENTS}

The authors are grateful to Cepheid for providing, 50\% discounted, Xpert reagents used for non-routine tests.

\section{REFERENCES}

1 Centers for Disease Control and Prevention. Reported Tuberculosis in United States, 2005. Atlanta, US Department of Health and Human Services, 2006.

2 European Centre for Disease Prevention and Control. World Health Organization Regional Office for Europe. Tuberculosis Surveillance in Europe 2009. Stockholm, European Centre for Disease Prevention and Control, 2011.

3 Centers for Disease Control and Prevention. Updated guidelines for the use of nucleic acid amplification tests in the diagnosis of tuberculosis. MMWR 2009; 58: 7-10.

4 World Health Organization. Report of the Tenth Meeting WHO Strategic and Technical Advisory Group for Tuberculosis (STAGTB), 27-29 September 2010. Geneva, World Health Organization, 2010.

5 Boehme CC, Nicol MP, Nabeta P, et al. Feasibility, diagnostic accuracy, and effectiveness of decentralised use of the Xpert MTB/ RIF test for diagnosis of tuberculosis and multidrug resistance: a multicentre implementation study. Lancet 2011; 377: 1495-1505.

6 Boehme CC, Nabeta P, Hillemann D, et al. Rapid molecular detection of tuberculosis and rifampin resistance. $N$ Engl J Med 2010; 363: 1005-1015.

7 Clinical and Laboratory Standards Institute. Laboratory Detection and Identification of Mycobacteria; Approved Guideline. Wayne, Clinical and Laboratory Standards Institute, 2008.

8 Tattevin P, Casalino E, Fleury L, et al. The validity of medical history, classic symptoms, and chest radiographs in predicting pulmonary tuberculosis - derivation of a pulmonary tuberculosis prediction model. Chest 1999; 115: 1248-1253.

9 Wisnivesky JP, Kaplan J, Henschke C, et al. Evaluation of clinical parameters to predict Mycobacterium tuberculosis in inpatients. Arch Intern Med 2000; 160: 2471-2476.

10 Davidoff F. Standing statistic right side up. Ann Intern Med 1999; 130: 1019-1021.

11 Hillemann D, Rusch-Gerdes S, Boehme C, et al. Rapid molecular detection of extrapulmonary tuberculosis by the automated GeneXpert MTB/RIF system. J Clin Microbiol 2011; 49: 1202-1205.

12 Vadwai V, Boehme C, Nabeta P, et al. Xpert MTB/RIF: a new pillar in diagnosis of extrapulmonary tuberculosis? J Clin Microbiol 2011; 49: 2540-2545.

13 Causse M, Ruiz P, Gutiérrez Aroca JB, et al. Comparison of two molecular methods for rapid diagnosis of extrapulmonary tuberculosis. J Clin Microbiol 2011; 49: 3065-3067.

14 Ligthelm LJ, Nicol MP, Hoek KG, et al. Xpert MTB/RIF for the rapid diagnosis of tuberculous lymphadenitis from fine needle aspiration biopsy specimens. J Clin Microbiol 2011; 49: 3967-3970.

15 Laraque F, Griggs A, Slopen M, et al. Performance of nucleic acid amplification tests for diagnosis of tuberculosis in a large urban setting. Clin Infect Dis 2009; 49: 46-54. 
16 Piersimoni C, Bornigia S, Gherardi G. Performance of a commercial nucleic acid amplification test with extrapulmonary specimens for the diagnosis of tuberculosis. Eur J Clin Microbiol Infect Dis 2012; 31: 287-293.

17 Armand S, Vanhuls P, Delcroix G, et al. Comparison of the Xpert MTB/RIF test with an IS6110-TaqMan real-time PCR assay for direct detection of Mycobacterium tuberculosis in respiratory and nonrespiratory specimens. J Clin Microbiol 2011; 49: 1772-1776.

18 Friedrich SO, Venter A, Kayigire XA, et al. Suitability of Xpert MTB/RIF and Genotype MTBDRplus for patient selection for a tuberculosis clinical trial. J Clin Microbiol 2011; 49: 2827-2831.

19 Helb D, Jones M, Story E, et al. Rapid detection of Mycobacterium tuberculosis and rifampin resistance by use of on-demand, nearpatient technology. J Clin Microbiol 2010; 48: 229-237.
20 Marlowe EM, Novak-Weekley SM, Cumpio J, et al. Evaluation of the Cepheid Xpert MTB/RIF assay for direct detection of Mycobacterium tuberculosis complex in respiratory specimens. J Clin Microbiol 2011; 49: 1621-1623.

21 Moure R, Munoz L, Torres $\mathrm{M}$, et al. Rapid detection of Mycobacterium tuberculosis complex and rifampin resistance in smear-negative clinical samples by use of an integrated real-time PCR method. J Clin Microbiol 2011; 49: 1137-1139.

22 Nicol MP, Workman L, Isaacs W, et al. Accuracy of the Xpert MTB/RIF test for the diagnosis of pulmonary tuberculosis in children admitted to hospital in Cape Town, South Africa: a descriptive study. Lancet Infect Dis 2011; 11: 819-824.

23 Theron G, Peter J, van Zyl-Smit R, et al. Evaluation of the Xpert MTB/ RIF assay for the diagnosis of pulmonary tuberculosis in a high HIV prevalence setting. Am J Respir Crit Care Med 2011; 184: 132-140. 